\title{
Polyethylenimine Nanogels Incorporated with Ultrasmall Iron Oxide Nanoparticles and Doxorubicin for MR Imaging-Guided Chemotherapy of Tumors
}

Yu Zou, Du Li, Yue Wang, Zhijun Ouyang, Yucheng Peng, Helena Tomás, Jindong Xia, João Rodrigues, Mingwu Shen,* and Xiangyang Shi*

Cite This: Bioconjugate Chem. 2020, 31, 907-915

Read Online

ABSTRACT: Development of versatile nanoplatforms for cancer theranostics remains a hot topic in the area of nanomedicine. We report here a general approach to create polyethylenimine (PEI)-based hybrid nanogels (NGs) incorporated with ultrasmall iron oxide $\left(\mathrm{Fe}_{3} \mathrm{O}_{4}\right)$ nanoparticles (NPs) and doxorubicin for $T_{1}$-weighted $\mathrm{MR}$ imagingguided chemotherapy of tumors. In this study, PEI NGs were first prepared using an inverse emulsion approach along with Michael addition reaction to cross-link the NGs, modified with citric acidstabilized ultrasmall $\mathrm{Fe}_{3} \mathrm{O}_{4}$ NPs through 1-ethyl-3-(3-(dimethylamino)propyl) carbodiimide hydrochloride (EDC) coupling, and physically loaded with anticancer drug doxorubicin (DOX). The formed hybrid NGs possess good water dispersibility and colloidal stability, excellent DOX loading efficiency (51.4\%), $\mathrm{pH}$-dependent release profile of DOX

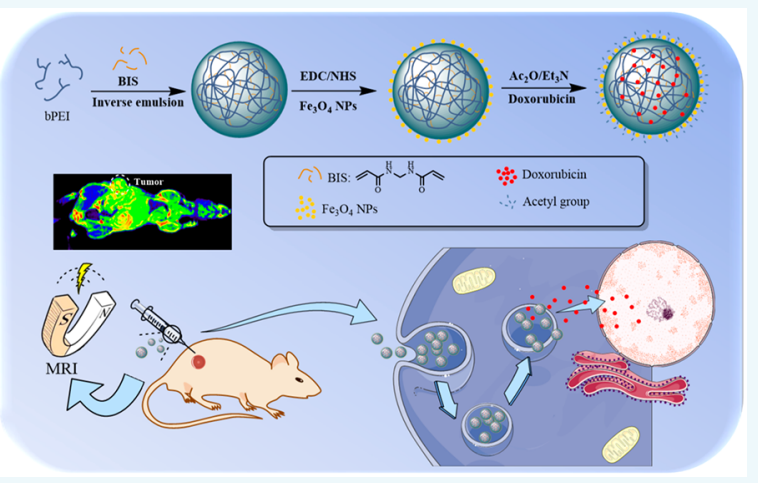
with an accelerated release rate under acidic $\mathrm{pH}$, and much higher $r_{1}$ relaxivity $\left(2.29 \mathrm{mM}^{-1} \mathrm{~s}^{-1}\right)$ than free ultrasmall $\mathrm{Fe}_{3} \mathrm{O}_{4} \mathrm{NPs}\left(1.15 \mathrm{mM}^{-1} \mathrm{~s}^{-1}\right)$. In addition, in contrast to the drug-free NGs that possess good cytocompatibility, the DOX-loaded hybrid NGs display appreciable therapeutic activity and can be taken up by cancer cells in vitro. With these properties, the developed hybrid NGs enabled effective inhibition of tumor growth under the guidance of $T_{1}$-weighted $M R$ imaging. The developed hybrid NGs may be applied as a versatile nanoplatform for MR imaging-guided chemotherapy of tumors.

\section{INTRODUCTION}

Nanomedicine remains a challenging field for cancer theranostics. To realize effective cancer theranostics, it is crucial to develop a variety of different nanoplatforms that can combine both therapeutic and diagnostic elements within the same platform. There are a myriad of nanomaterials including but not limited to nanotubes, ${ }^{1,2}$ nanofibers, ${ }^{3}$ quantum dots, ${ }^{4,5}$ micelles, $^{6}$ dendrimers, ${ }^{7-9}$ and nanogels $(\mathrm{NGs})^{10,11}$ that have been used in nanomedicine. For effective integration of theranostic functionalities, the nanoplatform incorporated with theranostic elements ${ }^{12-14}$ should possess reasonable colloidal stability, improved tumor penetration, and desired drug release kinetics after reaching the tumor site, thereby rendering effective tumor theranostics.

Among the commonly reported nanoplatforms, NGs possessing the properties of both bulk gels and nanoparticles (NPs) have received considerable attention for cancer imaging and therapy. ${ }^{15-18}$ Compared to other nanoplatforms, NGs have good fluidity, softness, and deformability, enabling better tumor penetration and more significant uptake by cancer cells than solid particles. ${ }^{19,20}$ In addition, NGs with spherical or quasi-spherical shapes and flexible skeletons can provide hydrophilic or amphiphilic surface and a huge amount of functional groups for theranostic components loading. ${ }^{21-23}$ Moreover, the internal cavities within three-dimensional structure of NGs enable effective encapsulation of NPs for various imaging applications. ${ }^{24,25}$ For instance, $\operatorname{poly}(\gamma$-glutamic acid) NGs can be loaded with iron oxide $\mathrm{NPs}^{26}$ and gold NPs, ${ }^{24}$ respectively, through an in situ cross-linking strategy for tumor magnetic resonance (MR) and computed tomography imaging.

Various methods, such as interfacial polymerization, ${ }^{27}$ precipitation polymerization, ${ }^{28}$ emulsification ${ }^{29-31}$ and solvent evaporation, $^{32}$ have been introduced for NGs synthesis. Among them, an inverse mini-emulsion method possesses advantages of easy preparation using functional polymers to

Received: January 20, 2020

Revised: February 24, 2020

Published: February 25, 2020 


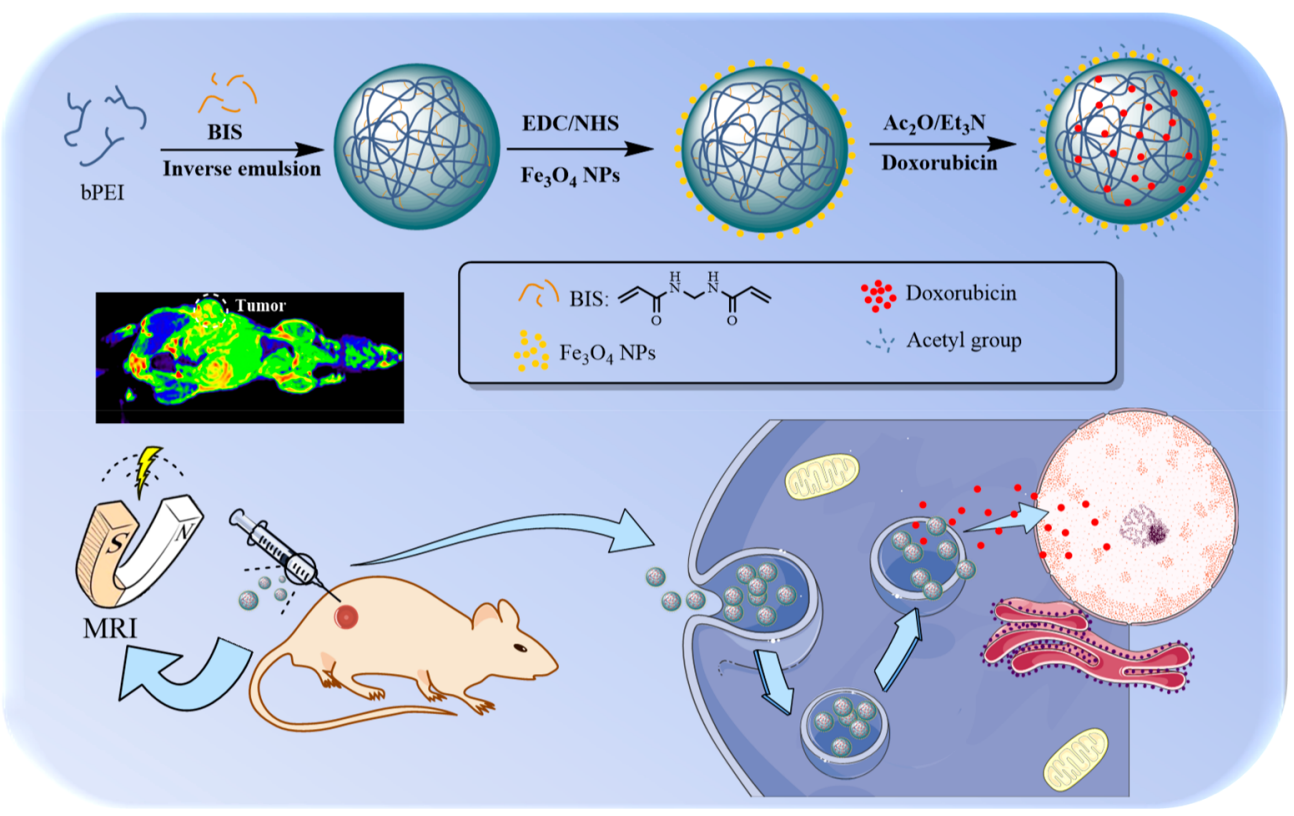

Figure 1. Schematic representation of the synthesis of $\mathrm{Fe}_{3} \mathrm{O}_{4} / \mathrm{PEI}-\mathrm{Ac}$ NGs/DOX complexes for MR imaging-guided chemotherapy of tumors.

form NGs having functional surface groups and control of size and cross-linking density. Recently, Pich and co-workers reported the creation of $N, N^{\prime}$-methylene-bis(acrylamide) (BIS)-cross-linked poly(allylamine) hydrochloride (PAH) NGs with abundant surface amine groups through an inverse mini-emulsion polymerization method. ${ }^{33}$ The formed PAH NGs were able to be further functionalized through the NG surface PAH amines. In another study, the same group reported the use of polymer NGs as a carrier to load anticancer drug DOX for enhance delivery applications. ${ }^{34}$ These studies highlight the importance to develop advanced functional NGbased platforms for drug delivery applications. However, only a few studies concern the development of NG-based platform for theranostic applications.

Recently, ultrasmall iron oxide $\left(\mathrm{Fe}_{3} \mathrm{O}_{4}\right)$ nanoparticles (NPs) with a size smaller than $5 \mathrm{~nm}$ have received immense interest for cancer theranostic applications due to the advantages of $T_{1}$ weighted MR imaging capacity, biocompatibility, easy synthesis, and tunable manipulation. ${ }^{35-37}$ For instance, ultrasmall $\mathrm{Fe}_{3} \mathrm{O}_{4} \mathrm{NPs}$ can be surface modified with zwitterions to have prolonged blood circulation time for enhanced tumor MR imaging, ${ }^{38}$ can be surface modified with targeting ligand folic acid via a poly(ethylene glycol) spacer for targeted tumor MR imaging, ${ }^{39}$ and can be incorporated within gold nanostars with near-infrared absorption feature for dual-mode MR/photoacoustic imaging-guided tumor photothermal therapy. ${ }^{40}$ These prior studies related to functional NGs and ultrasmall $\mathrm{Fe}_{3} \mathrm{O}_{4}$ NPs prompt us to hypothesize that polyethylenimine (PEI) NGs may be synthesized via inverse mini-emulsion and be used as a platform to incorporate ultrasmall $\mathrm{Fe}_{3} \mathrm{O}_{4} \mathrm{NPs}$ and anticancer drug for MR imaging-guided chemotherapy of tumors.

In this study, we first prepared PEI NGs using an inverse mini-emulsion method and cross-linked them with BIS via Michael addition reaction. The synthesized NGs with abundant surface amines were covalently conjugated with citric acid-stabilized ultrasmall $\mathrm{Fe}_{3} \mathrm{O}_{4}$ NPs synthesized via solvothermal method according to our previous report ${ }^{41}$ through 1-ethyl-3-(3-(dimethylamino)propyl) carbodiimide hydrochloride (EDC) coupling, followed by acetylation of the remaining NG surface amines and physical encapsulation of an anticancer drug doxorubicin (DOX) (Figure 1). The generated $\mathrm{Fe}_{3} \mathrm{O}_{4} /$ PEI-Ac NGs/DOX complexes were systematically characterized via different techniques in terms of their structure, composition, morphology, stability, release kinetics, and $r_{1}$ relaxivity. We then assessed the cytocompatibility of drug-free NGs, in vitro cellular uptake capacity and therapeutic activity, and in vivo MR imaging-guided tumor chemotherapy. To the best of our knowledge, this is the very first example to develop PEI-based NG systems for MR imaging-guided tumor chemotherapy.

\section{RESULTS AND DISCUSSION}

Synthesis and Characterization of $\mathrm{Fe}_{3} \mathrm{O}_{4} / \mathrm{PEI}-\mathrm{Ac}$ NGs. In this work, through the inverse mini-emulsion method and BIS-mediated cross-linking, ${ }^{11}$ PEI NGs with abundant amines were prepared, surface functionalized with ultrasmall $\mathrm{Fe}_{3} \mathrm{O}_{4}$ NPs through EDC coupling, and finally acetylated to neutralize the remaining NG surface amines (Figure 1). The prepared NGs and the intermediate products were systematically characterized through different methods.

First, the surface potential of NGs after each step of surface modification was monitored (Table S1). The pristine PEI NGs have a surface potential of $38.9 \pm 0.62 \mathrm{mV}$ due to their abundant surface amine groups. After conjugation with negatively charged citric acid-stabilized ultrasmall $\mathrm{Fe}_{3} \mathrm{O}_{4} \mathrm{NPs}$ $(-37.9 \pm 1.35 \mathrm{mV})$, the formed $\mathrm{Fe}_{3} \mathrm{O}_{4} /$ PEI NGs display decreased surface potential of $29.3 \pm 0.88 \mathrm{mV}$. Final acetylation of the $\mathrm{Fe}_{3} \mathrm{O}_{4} / \mathrm{PEI} \mathrm{NGs}$ led to the further decreased surface potential of the $\mathrm{Fe}_{3} \mathrm{O}_{4} /$ PEI-Ac NGs $(13.3 \pm 1.15 \mathrm{mV})$.

Likewise, the hydrodynamic size of the NGs after stepwise modification was measured by dynamic light scattering (DLS, Table S1). Pristine PEI NGs were measured to have a hydrodynamic size of $180.38 \pm 5.48 \mathrm{~nm}$. After covalent conjugation with ultrasmall $\mathrm{Fe}_{3} \mathrm{O}_{4} \mathrm{NPs}$ having a hydrodynamic size of $27.5 \pm 3.2 \mathrm{~nm}$ (dry single particle size was $2.8 \mathrm{~nm}^{38}$ as measured by transmission electron microscopy), the formed $\mathrm{Fe}_{3} \mathrm{O}_{4} /$ PEI NGs have an increased size of $251.4 \pm 6.34 \mathrm{~nm}$. 
The last step of acetylation led to a further increased hydrodynamic size of the $\mathrm{Fe}_{3} \mathrm{O}_{4} /$ PEI-Ac NGs $(263.8 \pm 3.81$ $\mathrm{nm})$. Furthermore, to assess the long-term colloidal stability of the NGs, we monitored the hydrodynamic sizes of both the pristine PEI NGs and the final $\mathrm{Fe}_{3} \mathrm{O}_{4} / \mathrm{PEI}$-Ac NGs dispersed in water via DLS (Figure S1). Clearly, the hydrodynamic sizes of both NGs do not seem to have any appreciable changes for at least 25 days, indicating their good colloidal stability.

Atomic force microscopy (AFM) was used to observe the morphology of the as-synthesized $\mathrm{Fe}_{3} \mathrm{O}_{4}$ /PEI-Ac NGs. As shown in Figure 2a,b, both PEI NGs and $\mathrm{Fe}_{3} \mathrm{O}_{4} /$ PEI-Ac NGs
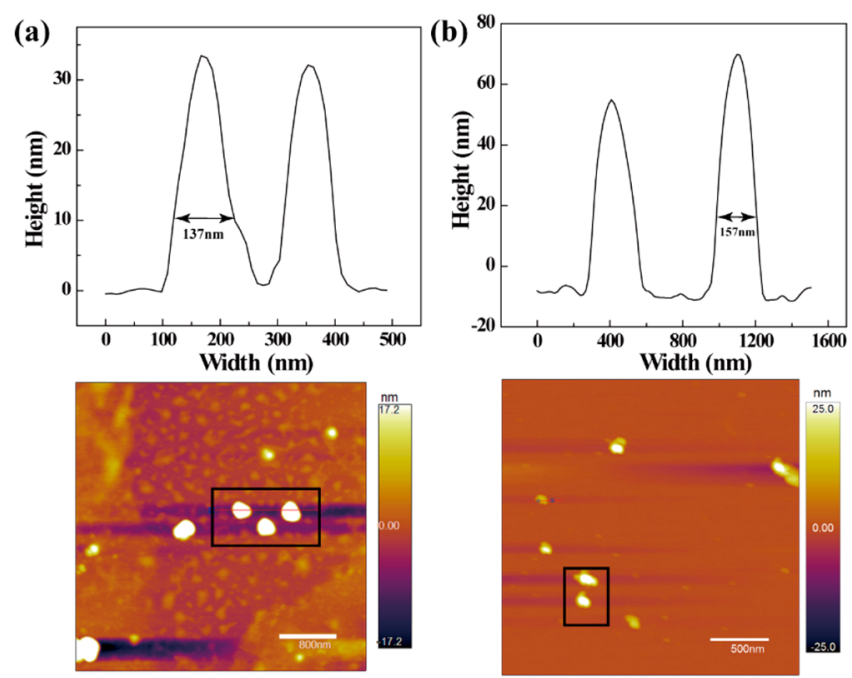

(c)

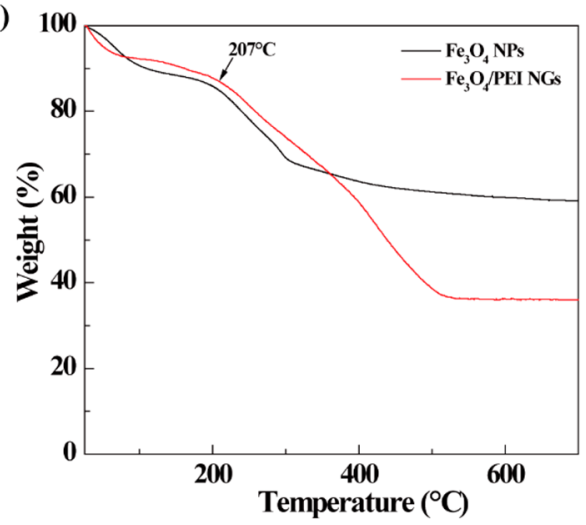

Figure 2. AFM images of (a) PEI NGs and (b) $\mathrm{Fe}_{3} \mathrm{O}_{4} / \mathrm{PEI}$-Ac NGs. (c) TGA curve of ultrasmall $\mathrm{Fe}_{3} \mathrm{O}_{4} \mathrm{NPs}$ and $\mathrm{Fe}_{3} \mathrm{O}_{4} /$ PEI NGs.

exhibit a uniform spherical shape. The size of pristine PEI NGs was measured to be $137 \mathrm{~nm}$. After conjugation with ultrasmall $\mathrm{Fe}_{3} \mathrm{O}_{4} \mathrm{NPs}$ and surface leftover amine acetylation, the size of the $\mathrm{Fe}_{3} \mathrm{O}_{4} /$ PEI-Ac NGs increased to $157 \mathrm{~nm}$. As expected, the size of the $\mathrm{Fe}_{3} \mathrm{O}_{4} / \mathrm{PEI}$-Ac NGs measured by AFM is smaller than that measured by DLS. This is likely since DLS analyzes the swollen state of the $\mathrm{Fe}_{3} \mathrm{O}_{4} / \mathrm{PEI}-\mathrm{Ac} \mathrm{NGs}$ in aqueous solution that may have a certain degree of aggregation, while AFM detects the single NGs in a dried state. Overall, the surface potential, DLS, and AFM measurements collectively confirmed the success of the preparation of the $\mathrm{Fe}_{3} \mathrm{O}_{4} / \mathrm{PEI}$-Ac NGs.

Thermogravimetric analysis (TGA) was used to quantify the grafting percentage of ultrasmall $\mathrm{Fe}_{3} \mathrm{O}_{4} \mathrm{NPs}$ onto the surface of PEI NGs (Figure 2c). Owing to the excellent thermal stability of PEI NGs, the weight of $\mathrm{Fe}_{3} \mathrm{O}_{4} /$ PEI NGs remains stable under the temperature of $207{ }^{\circ} \mathrm{C}$. By subtracting the weight loss of ultrasmall $\mathrm{Fe}_{3} \mathrm{O}_{4} \mathrm{NPs}(29.3 \%)$ from that of the $\mathrm{Fe}_{3} \mathrm{O}_{4}$ / PEI NGs (55.3\%), we were able to calculate the percentage of the PEI content of the hybrid NGs at $26.0 \%$. Consequently, the loading percentage of ultrasmall $\mathrm{Fe}_{3} \mathrm{O}_{4} \mathrm{NPs}$ onto the PEI NGs was calculated to be $74.0 \%$.

$\mathrm{Fe}_{3} \mathrm{O}_{4} \mathrm{NPs}$ with a size smaller than $5 \mathrm{~nm}$ have been used as a contrast agent for $T_{1}$-weighted $\mathrm{MR}$ imaging. ${ }^{42}$ Here, the potential of the $\mathrm{Fe}_{3} \mathrm{O}_{4} /$ PEI-Ac NGs to be used for $T_{1}$-weighted $\mathrm{MR}$ imaging was explored through relaxometry studies (Figure 3). It is found that both citric acid-stabilized $\mathrm{Fe}_{3} \mathrm{O}_{4} \mathrm{NPs}$ and
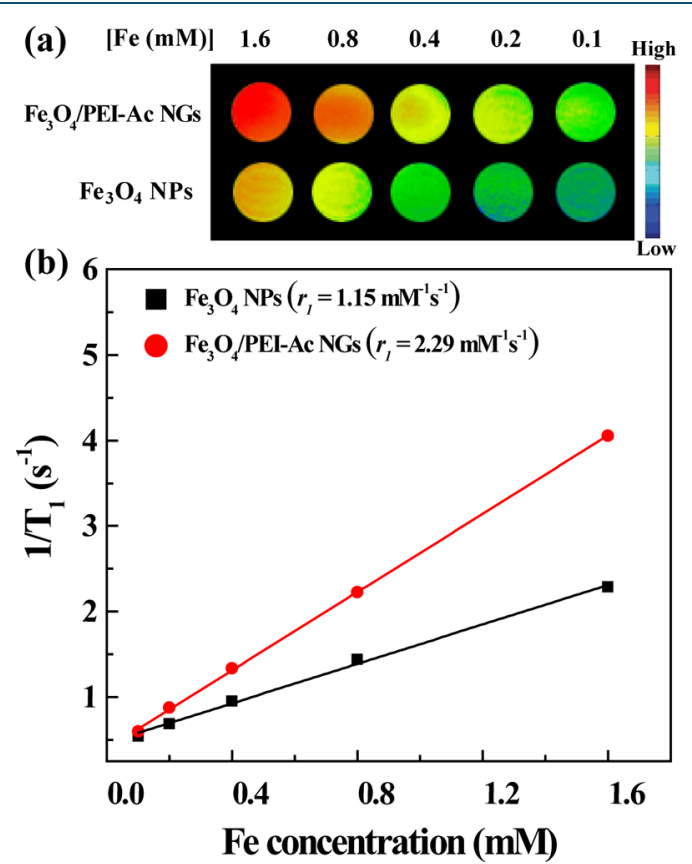

Figure 3. (a) Color $T_{1}$-weighted $\mathrm{MR}$ images of the ultrasmall $\mathrm{Fe}_{3} \mathrm{O}_{4}$ NPs and $\mathrm{Fe}_{3} \mathrm{O}_{4} /$ PEI-Ac NGs at an Fe concentration of 0.1, 0.2, 0.4, 0.8 , and $1.6 \mathrm{mM}$, respectively. The color bar from blue to red indicates the gradual increase of MR signal intensity. (b) Linear fitting of $1 / T_{1}$ as a function of $\mathrm{Fe}$ concentration for the two different materials.

$\mathrm{Fe}_{3} \mathrm{O}_{4} /$ PEI-Ac NGs can improve MR signal intensity in the $T_{1}$ weighted $\mathrm{MR}$ images with the increase of $\mathrm{Fe}$ concentration (Figure 3a). By linearly fitting the $T_{1}$ relaxation $\left(1 / T_{1}\right)$ as a function of $\mathrm{Fe}$ concentration (Figure $3 \mathrm{~b}$ ), the $r_{1}$ relaxivities of ultrasmall $\mathrm{Fe}_{3} \mathrm{O}_{4}$ NPs and $\mathrm{Fe}_{3} \mathrm{O}_{4} /$ PEI-Ac NGs were measured to be $1.15 \mathrm{mM}^{-1} \mathrm{~s}^{-1}$ and $2.29 \mathrm{mM}^{-1} \mathrm{~s}^{-1}$, respectively. The higher $r_{1}$ relaxivity of the $\mathrm{Fe}_{3} \mathrm{O}_{4} /$ PEI-Ac NGs than that of free ultrasmall $\mathrm{Fe}_{3} \mathrm{O}_{4}$ NPs could be ascribed to the enlarged molecular volume of the particles within the PEI NGs with prolonged rotational correlation time, thus presenting an increased $r_{1}$ relaxivity and improved MR imaging sensitivity. ${ }^{43}$

Drug Encapsulation and Release Kinetics. Since the NGs possess many PEI polymers with internal hydrophobic cavities that are suitable for DOX encapsulation, ${ }^{44}$ we physically loaded DOX within the NGs. The DOX loading efficiency and capacity within the hybrid $\mathrm{Fe}_{3} \mathrm{O}_{4}$ /PEI-Ac NGs were measured via UV-vis spectroscopy using the standard calibration curve of DOX absorption (at $480 \mathrm{~nm}$ )/concentration in phosphate buffered saline (PBS) solution. We optimized ratios between DOX and $\mathrm{Fe}_{3} \mathrm{O}_{4}$ /PEI-Ac NGs during the drug loading process to achieve the best loading efficiency 
and loading capacity. At different DOX/NGs mass ratios (1:2, $1: 1$, or $2: 1)$, the loading efficiency and loading capacity were measured (Table S2). It can be found that at the DOX/NGs mass ratio of $1: 1$, the best DOX loading percentage (21.9\%) can be achieved, while at the DOX/NGs mass ratio of $2: 1$, the maximum drug loading efficiency $(51.4 \%)$ can be observed. Based on these results, we selected 2:1 as the optimal ratio for the subsequent experiments.

The in vitro release study of DOX from the $\mathrm{Fe}_{3} \mathrm{O}_{4} / \mathrm{PEI}-\mathrm{Ac}$ NGs/DOX complexes was performed in phosphate buffer within different $\mathrm{pH}$ environments $(\mathrm{pH} 5.5$ and $\mathrm{pH} 7.4)$ at 37 ${ }^{\circ} \mathrm{C}$ (Figure 4). Apparently, the release of DOX from the

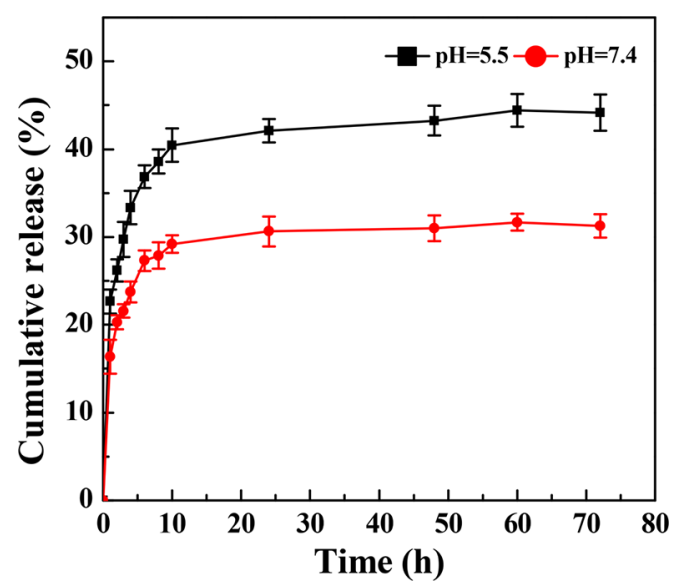

Figure 4. Cumulative release of DOX from the $\mathrm{Fe}_{3} \mathrm{O}_{4} / \mathrm{PEI}-\mathrm{Ac}$ NGs/ DOX complexes under different $\mathrm{pHs}$.
$\mathrm{Fe}_{3} \mathrm{O}_{4} / \mathrm{PEI}$-Ac NGs/DOX complexes shows a sustained manner under both pHs. However, the cumulative release of DOX from the complexes was $44.1 \pm 2.05 \%$ at $72 \mathrm{~h}$ in an acidic environment $(\mathrm{pH}=5.5)$, which was much higher than that $(31.3 \pm 1.32 \%)$ in a physiological environment $(\mathrm{pH}=7.4)$ at the same time point. These results indicate that the DOX release from the hybrid $\mathrm{NGs}$ is $\mathrm{pH}$-sensitive with a higher release speed under a slightly acidic environment than under a physiological $\mathrm{pH}$ environment. The fast release of DOX under the slightly acidic $\mathrm{pH}$ is certainly beneficial for chemotherapy of tumors with a slightly acidic microenvironment $(\mathrm{pH} 5.5-$ 6.5). The rapid DOX release from the NGs could be due to the fact that DOX is much more water-soluble under acidic $\mathrm{pH}$ than under physiological $\mathrm{pH}$, in agreement with the literature. $^{45}$

Therapeutic Efficacy of $\mathrm{Fe}_{3} \mathrm{O}_{4} / \mathrm{PEI}-\mathrm{Ac} \mathrm{NGs} / \mathrm{DOX}$ Complexes. We next assessed the therapeutic efficacy of the $\mathrm{Fe}_{3} \mathrm{O}_{4}$ /PEI-Ac NGs/DOX complexes in vitro through CCK-8 cell viability assay (Figure $5 \mathrm{a}$ ). Free $\mathrm{DOX} \cdot \mathrm{HCl}$ and drug-free $\mathrm{Fe}_{3} \mathrm{O}_{4} /$ PEI-Ac NGs were also tested for comparison. Clearly, drug-free $\mathrm{Fe}_{3} \mathrm{O}_{4}$ /PEI-Ac NGs display an excellent cytocompatibility and the tested murine mammary carcinoma 4T1 cells display a viability around $100 \%$ after the treatment of NGs at a concentration up to $40 \mu \mathrm{g} / \mathrm{mL}$ for $24 \mathrm{~h}$. In contrast, both free $\mathrm{DOX} \cdot \mathrm{HCl}$ and the NGs/DOX complexes display apparent therapeutic efficacy to inhibit the growth of cancer cells. The lower therapeutic efficacy of the NGs/DOX complexes than that of free $\mathrm{DOX} \cdot \mathrm{HCl}$ should be due to the slow release of DOX from the $\mathrm{Fe}_{3} \mathrm{O}_{4} / \mathrm{PEI}$-Ac NGs/DOX, in agreement with the literature. ${ }^{45}$ Our data suggest that the $\mathrm{Fe}_{3} \mathrm{O}_{4}$ /PEI-Ac NGs have a good cytocompatibility and can be used as a carrier system for drug delivery to inhibit cancer cells in vitro.

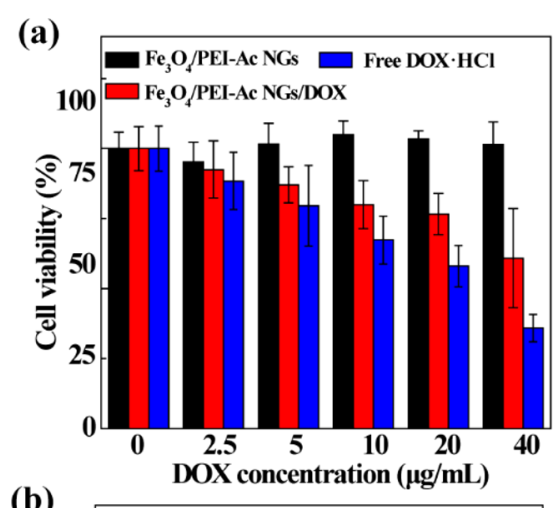

(c)

(b)
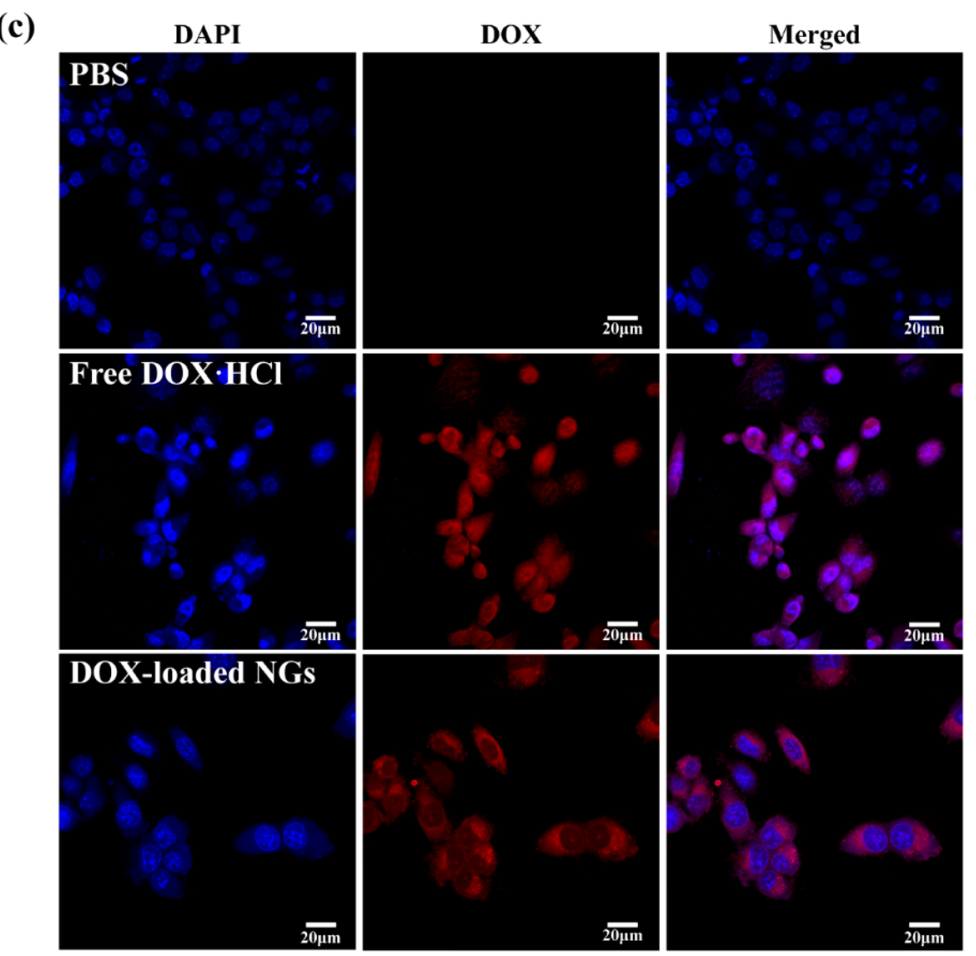

Figure 5. (a) CCK-8 assay of $4 \mathrm{~T} 1$ cell viability after they were treated with $\mathrm{Fe}_{3} \mathrm{O}_{4} / \mathrm{PEI}$-Ac NGs/DOX, free DOX.HCl, and $\mathrm{Fe} \mathrm{O}_{3} / \mathrm{PEI}-\mathrm{Ac} \mathrm{NGs}$ at different DOX concentrations for $24 \mathrm{~h}$. The $4 \mathrm{~T} 1$ cells treated with PBS were used as a control. (b) Mean fluorescence of cells treated with $\mathrm{Fe}_{3} \mathrm{O}_{4} /$ PEI-Ac NGs/DOX as a function of DOX concentration. (c) Confocal microscopic images of 4T1 cells after treatment for $4 \mathrm{~h}$ with the free DOX. $\mathrm{HCl}$ and $\mathrm{Fe}_{3} \mathrm{O}_{4} / \mathrm{PEI}-\mathrm{Ac} \mathrm{NGs} / \mathrm{DOX}$ at a DOX concentration of $10 \mu \mathrm{g} / \mathrm{mL}$. The $4 \mathrm{~T} 1$ cells treated with PBS were used as a control. 

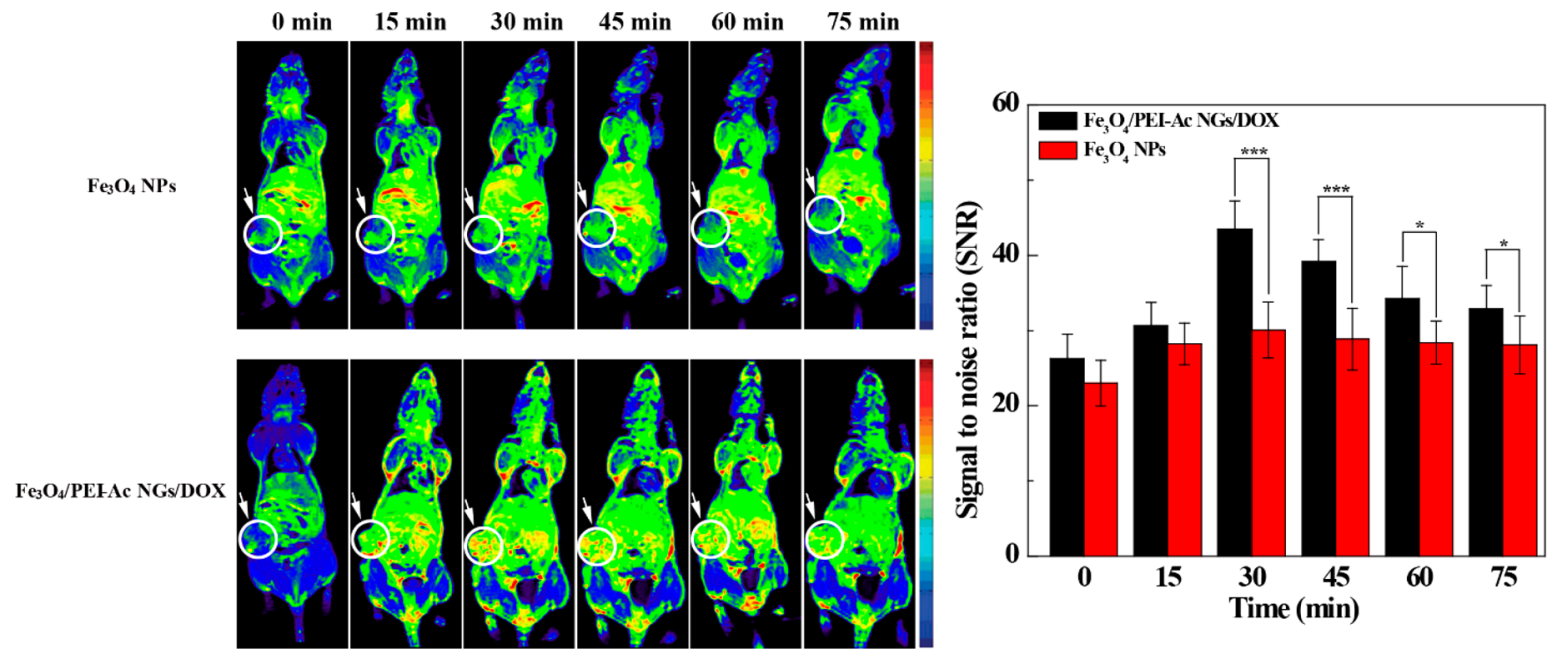

Figure 6. In vivo $T_{1}$-weighted $\mathrm{MR}$ images (a) and MR signal-to-noise ratio (SNR) (b) of the xenografted $4 \mathrm{~T} 1$ tumors before and at different time points post intravenous injection of the free ultrasmall $\mathrm{Fe}_{3} \mathrm{O}_{4} \mathrm{NPs}$ or the $\mathrm{Fe}_{3} \mathrm{O}_{4} / \mathrm{NGs}-\mathrm{Ac} \mathrm{NGs} / \mathrm{DOX}$ complexes $(\mathrm{Fe}$ mass $=150 \mu \mathrm{g}$, in $0.2 \mathrm{~mL}$ PBS for each mouse).

(a)

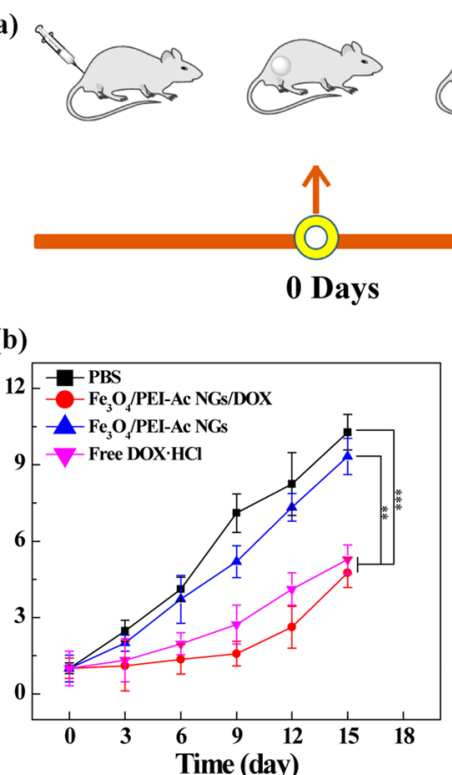

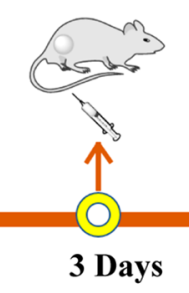

(c)

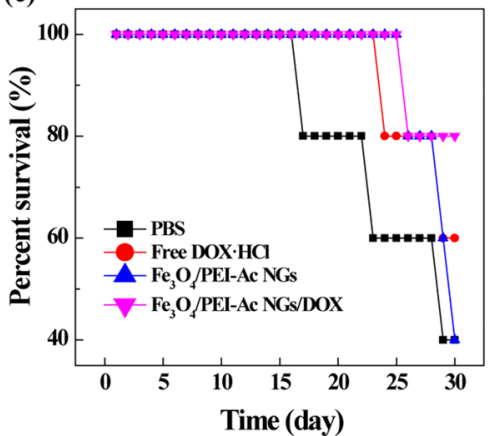

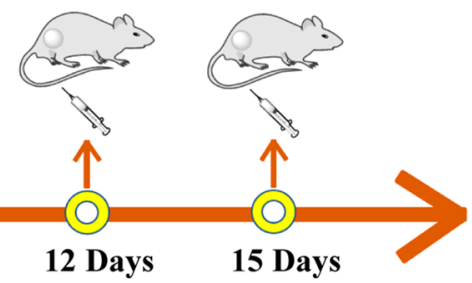

(d)

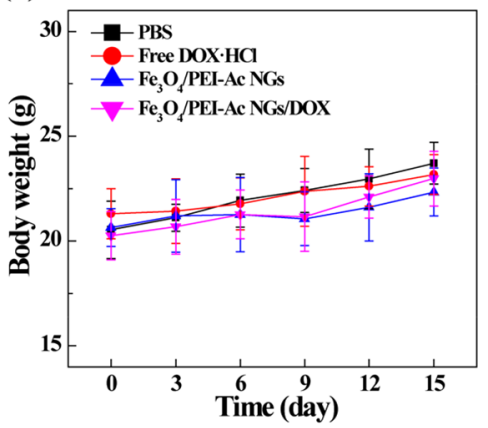

Figure 7. (a) Schematic diagram of the treatment process of the tumor-bearing mice in vivo. (b) Relative tumor volume of mice after different treatments for 15 days. (c) Survival rate of the mice in each group during 30 days treatment. (d) Body weight of mice after different treatments for 15 days.

To check the cellular uptake of DOX-loaded NGs, flow cytometry assay was performed (Figure $5 \mathrm{~b}$ ). As shown in Figure S2, the right-shifted histograms indicate that the cells are able to take up the NGs to display DOX-associated fluorescence signal. With the increase of DOX concentration, the cell fluorescence histogram displays a more apparent rightshift trend, indicating that there are more NGs taken up by cells. This trend can be further proven by plotting the mean fluorescence of cells as a function of DOX concentration of the NGs (Figure $5 \mathrm{~b}$ ). At the DOX concentration of $20 \mu \mathrm{g} / \mathrm{mL}$, the mean fluorescence signal of cells is 37 times higher than that at the DOX concentrations of $1 \mu \mathrm{g} / \mathrm{mL}$, and 2.5 times higher than that at the DOX concentration of $5 \mu \mathrm{g} / \mathrm{mL}$.

Confocal laser scanning microscopy was also used to visually confirm the cellular uptake of the DOX-loaded NGs (Figure $5 c)$. The red fluorescence of DOX can only be detected in the cytoplasm of cells treated with free $\mathrm{DOX} \cdot \mathrm{HCl}$ and $\mathrm{Fe}_{3} \mathrm{O}_{4} / \mathrm{PEI}-$
Ac NGs/DOX after $4 \mathrm{~h}$ incubation of cells, while the control group treated with PBS does not display any red fluorescent signals. Free DOX $\cdot \mathrm{HCl}$ group shows purple nuclei and red cytoplasm, implying that free $\mathrm{DOX} \cdot \mathrm{HCl}$ can enter cell nuclei with blue staining. For cells treated with the $\mathrm{Fe}_{3} \mathrm{O}_{4} / \mathrm{PEI}-\mathrm{Ac}$ NGs/DOX complexes, both blue and red fluorescence can be spotted, and the DOX-associated red fluorescence mainly appears in the cytoplasm. It takes time for DOX to be released from the $\mathrm{Fe}_{3} \mathrm{O}_{4} /$ PEI-Ac NGs/DOX complexes to enter the cell nuclei.

To further confirm the NG taken up by cells, we analyzed the cellular Fe concentration using inductively coupled plasmaoptical emission spectroscopy (ICP-OES) (Figure S3). After treatment with the $\mathrm{Fe}_{3} \mathrm{O}_{4}$ /PEI-Ac NGs/DOX complexes at different DOX concentrations ranging from 0 to $90 \mu \mathrm{g} / \mathrm{mL}$ for $24 \mathrm{~h}$, the $4 \mathrm{~T} 1$ cells were digested by aqua regia and subjected to ICP-OES assay. Obviously, the cellular Fe uptake increases 
with the increase of the DOX concentration, which is consistent with the results obtained from confocal laser scanning microscopy and flow cytometry assays.

In Vivo MR Imaging-Guided Antitumor Chemotherapy. Due to the excellent $r_{1}$ relaxivity of the ultrasmall $\mathrm{Fe}_{3} \mathrm{O}_{4} \mathrm{NPs}$ incorporated within the NGs, we tested the potential to use the as-prepared $\mathrm{Fe}_{3} \mathrm{O}_{4} / \mathrm{PEI}-\mathrm{Ac} \mathrm{NGs} / \mathrm{DOX}$ complexes as a contrast agent for $\mathrm{MR}$ imaging of tumors in vivo. For comparison, free ultrasmall $\mathrm{Fe}_{3} \mathrm{O}_{4}$ NPs were also tested under the same conditions. As shown in Figure 6, the MR signal intensity of tumor (as indicated in the white circles) increases first with the time post-injection, and then declines at 30 min post-injection. This suggests that free ultrasmall $\mathrm{Fe}_{3} \mathrm{O}_{4}$ $\mathrm{NPs}$ and the $\mathrm{Fe}_{3} \mathrm{O}_{4} /$ PEI-Ac NGs/DOX complexes can be enriched in the tumor site with blood circulation after intravenous injection possibly through the passive enhanced permeability and retention (EPR) effect. By quantitatively analyzing the tumor MR signal-to-noise ratio (SNR), we find that the MR SNR of $\mathrm{Fe}_{3} \mathrm{O}_{4} / \mathrm{PEI}$-Ac NGs/DOX group is much higher than that of free ultrasmall $\mathrm{Fe}_{3} \mathrm{O}_{4}$ NPs group at the same time points, possibly due to the larger size of NGs possessing a better tumor EPR effect than that of the free ultrasmall $\mathrm{Fe}_{3} \mathrm{O}_{4}$ NPs. At 30 min post-injection, the $\mathrm{Fe}_{3} \mathrm{O}_{4}$ / PEI-Ac NGs/DOX complexes can be gradually metabolized in vivo, leading to gradually decreased $\mathrm{Fe}$ concentration in the tumor site. These results suggest that the $\mathrm{Fe}_{3} \mathrm{O}_{4}$ /PEI-Ac NGs/ DOX complexes display an enhanced $\mathrm{MR}$ imaging performance than free ultrasmall $\mathrm{Fe}_{3} \mathrm{O}_{4}$ NPs.

We then checked the biodistribution of the $\mathrm{Fe}_{3} \mathrm{O}_{4} / \mathrm{PEI}-\mathrm{Ac}$ $\mathrm{NGs} / \mathrm{DOX}$ complexes in the major organs and tumor of mice at different time points post-administration to evaluate the metabolic pathway (Figure S4). At an earlier time point of 0.5 $\mathrm{h}$, spleen and tumor tissues display much high Fe uptake than other time points. After that, the intravenously injected $\mathrm{Fe}_{3} \mathrm{O}_{4} /$ PEI-Ac NGs/DOX complexes were taken up predominantly by spleen and lung and gradually cleared from kidney within $48 \mathrm{~h}$. It should be pointed that at $0.5 \mathrm{~h}$ post-injection, the tumor site displays a significantly high amount of Fe uptake, thus enabling effective MR imaging of tumors. These results suggest that the developed $\mathrm{Fe}_{3} \mathrm{O}_{4} /$ PEI-Ac NGs/DOX complexes could accumulate in tumor tissue via EPR passive targeting pathway and be safely excreted from the host animals.

Lastly, we evaluated the chemotherapeutic efficacy of the $\mathrm{Fe}_{3} \mathrm{O}_{4} /$ PEI-Ac NGs/DOX complexes to treat the xenografted $4 \mathrm{~T} 1$ breast tumor model. The treatment schedule of 4T1bearing $\mathrm{BALB} / \mathrm{c}$ mice is shown in Figure $7 \mathrm{a}$. The chemotherapy efficacy of tumor bearing mice was evaluated via monitoring the relative tumor volume change (Figure $7 \mathrm{~b}$ ). It can be seen that the treatment with both free DOX $\cdot \mathrm{HCl}$ drug and the DOX-loaded $\mathrm{Fe}_{3} \mathrm{O}_{4} / \mathrm{PEI}-\mathrm{Ac} \mathrm{NGs}$ are able to significantly inhibit the growth of tumors, and the $\mathrm{Fe}_{3} \mathrm{O}_{4}$ / PEI-Ac NGs/DOX group has a better tumor inhibition effect than free DOX.HCl group. On the contrary, the drug-free $\mathrm{Fe}_{3} \mathrm{O}_{4} /$ PEI-Ac NGs group exhibits the same tumor growth trend as the PBS control. Further, mice survival rate was used to evaluate the chemotherapy efficacy of tumors (Figure $7 \mathrm{c}$ ). Only mice treated with the $\mathrm{Fe}_{3} \mathrm{O}_{4} / \mathrm{PEI}-\mathrm{Ac} \mathrm{NG} / \mathrm{DOX}$ complexes maintain an $80 \%$ survival rate during a period of 30 days, while all mice treated with PBS and DOX-free $\mathrm{Fe}_{3} \mathrm{O}_{4}$ / PEI-Ac NGs maintain a $40 \%$ survival rate, and mice treated with free $\mathrm{DOX} \cdot \mathrm{HCl}$ have a survival rate of $60 \%$. Additionally, the body weights of mice treated with the $\mathrm{Fe}_{3} \mathrm{O}_{4}$ /PEI-Ac NGs/ DOX complexes do not change drastically (Figure $7 \mathrm{~d}$ ), like other groups showing the same body weight change with slightly increasing trend after 15 days of treatment. This suggests that the treatment of all groups does not seem to create apparent safety concerns. The biosafety of the $\mathrm{Fe}_{3} \mathrm{O}_{4} /$ PEI-Ac NGs/DOX complexes was further assessed through hematoxylin and eosin (H\&E) staining of the major organs of mice after intravenous injection for 30 days (Figure S5). Clearly, the injection of the $\mathrm{Fe}_{3} \mathrm{O}_{4}$ /PEI-Ac NGs/DOX complexes does not seem to cause any significant damages to major organs of mice, and there is no significant change in organ morphology when compared with the PBS control, free DOX $\cdot \mathrm{HCl}$, and DOX-free $\mathrm{Fe}_{3} \mathrm{O}_{4} / \mathrm{PEI}$-Ac NGs groups. Our results suggest that the developed $\mathrm{Fe}_{3} \mathrm{O}_{4} /$ PEI-Ac NGs/DOX complexes do not influence the major organs of mice, thus having a good biosafety profile.

\section{CONCLUSION}

In summary, we developed a unique method to generate hybrid PEI-based NGs for tumor theranostics. The pristine PEI NGs with a size of $137 \mathrm{~nm}$ can be formed through an inverse mini-emulsion approach along with BIS-mediated cross-linking. Due to the abundant surface amines, the PEI NGs can be modified with citric acid-stabilized ultrasmall $\mathrm{Fe}_{3} \mathrm{O}_{4}$ NPs through EDC coupling reaction and be further acetylated to neutralize the remaining surface amines. The generated hybrid $\mathrm{Fe}_{3} \mathrm{O}_{4}$ /PEI-Ac NGs can be loaded with anticancer drug DOX through physical encapsulation and release the loaded DOX in a $\mathrm{pH}$-dependent manner. Importantly, the covalent conjugation of ultrasmall $\mathrm{Fe}_{3} \mathrm{O}_{4}$ NPs onto the surface of PEI NGs rendered the platform with significantly improved $r_{1}$ relaxivity $\left(2.29 \mathrm{mM}^{-1} \mathrm{~s}^{-1}\right)$. With these properties along with the good cytocompatibility of the drug-free NGs and the noncompromised therapeutic efficacy in vitro, the developed $\mathrm{Fe}_{3} \mathrm{O}_{4} / \mathrm{PEI}$-Ac NGs/DOX complexes enabled $T_{1}$-weighted $\mathrm{MR}$ imaging-guided tumor chemotherapy. The developed hybrid NGs may be further linked with targeting ligands (e.g., folic acid, peptide, etc.) through the PEI NG surface amines to realize active targeting of a specific tumor/disease type, thus holding a great promise to be used as a versatile theranostic platform to treat other types of tumors or diseases.

\section{EXPERIMENTAL SECTION}

Synthesis of PEI NGs Modified with Ultrasmall $\mathrm{Fe}_{3} \mathrm{O}_{4} \mathrm{NPs}\left(\mathrm{Fe}_{3} \mathrm{O}_{4} / \mathrm{PEI}-\mathrm{Ac} \mathrm{NGs}\right)$. First, ultrasmall citratestabilized $\mathrm{Fe}_{3} \mathrm{O}_{4}$ NPs were prepared according to the literature. $^{21,39}$ To prepare PEI NGs, Span 80 (1.2 g) was dissolved in toluene $(60 \mathrm{~mL})$ under stirring at room temperature for $30 \mathrm{~min}$. PEI $(272 \mathrm{mg}$ ) and BIS (32 mg) were codissolved in $5 \mathrm{~mL}$ of water, dropwise added into the above solution under stirring for $120 \mathrm{~min}$, followed by emulsification of the mixture solution through sonication. After that, triethylamine as a catalyst was added into solution to initiate the BIS cross-linking of PEI under stirring at room temperature overnight. Lastly, the NGs were collected by centrifugation (13000 rpm, $15 \mathrm{~min}$ ) and redispersion in methanol for 3 times to remove toluene, dialyzed against water (2 L, 6 times) using a dialysis membrane with a molecular weight cutoff (MWCO) of 8000-14000 for 3 days, centrifuged to concentrate the dialysis liquid, and finally stored in water at $4{ }^{\circ} \mathrm{C}$ before use. 
To modify the NGs with ultrasmall $\mathrm{Fe}_{3} \mathrm{O}_{4} \mathrm{NPs}$, the citric acid-stabilized $\mathrm{Fe}_{3} \mathrm{O}_{4} \mathrm{NPs}(50 \mathrm{mg})$ were dispersed in water $(5$ $\mathrm{mL})$, activated by EDC (75 mg)/NHS (45 mg), and dropwise added into the solution of PEI NGs $(250 \mathrm{mg}, 25 \mathrm{~mL}$ in water) under magnetic stirring at room temperature overnight. The product was collected through centrifugation (13000 rpm, 15 min) and redispersion in water for 3 times. After that, the $\mathrm{Fe}_{3} \mathrm{O}_{4} /$ PEI NGs were obtained and stored in water at $4{ }^{\circ} \mathrm{C}$.

To render the $\mathrm{Fe}_{3} \mathrm{O}_{4} / \mathrm{PEI} \mathrm{NGs}$ with close to neutral surface charge, the NGs with surface PEI amines were acetylated by acetic anhydride. In brief, triethylamine $(134.1 \mu \mathrm{L})$ was dropwise added into the solution of the $\mathrm{Fe}_{3} \mathrm{O}_{4} /$ PEI NGs $(20$ $\mathrm{mg}, 2 \mathrm{~mL}$ ) under stirring for $30 \mathrm{~min}$. Then, acetic anhydride $(75.9 \mu \mathrm{L})$ was added into the mixture solution under stirring overnight. The product was purified by membrane dialysis (MWCO $=8000-14000)$ for 2 days, and then the final product was concentrated through centrifugation and stored at $4{ }^{\circ} \mathrm{C}$.

Drug Loading within and Release from the Hybrid NGs. The $\mathrm{Fe}_{3} \mathrm{O}_{4} /$ PEI-Ac NGs (10 mg) were dispersed in 1 $\mathrm{mL}$ of phosphate buffered saline (PBS). DOX. $\mathrm{HCl}$ was dissolved in $1 \mathrm{~mL}$ of water and mixed with the $\mathrm{Fe}_{3} \mathrm{O}_{4} / \mathrm{PEI}$ Ac NG solution at a DOX/NG mass ratio of $0.5,1$, or 2 , respectively under stirring at room temperature overnight. After that, the mixture solution was centrifuged (13000 rpm for $15 \mathrm{~min}$ ) to precipitate the drug-loaded NGs. The drugloaded NGs were washed with water for 3 times and the supernatants in each step (containing the noncomplexed free $\mathrm{DOX} \cdot \mathrm{HCl}$ ) were collected. The precipitate was redispersed in $2 \mathrm{~mL}$ of PBS and stored at $4{ }^{\circ} \mathrm{C}$. The supernatants were analyzed via UV-vis spectroscopy. The loading percentage of DOX in the NGs was calculated by dividing the loaded DOX (subtraction of the initial added DOX amount with that in the supernatants) by the mass of the DOX/NGs, while the loading efficiency was calculated by dividing the mass of loaded DOX by that of the initial total DOX.

The formed $\mathrm{Fe}_{3} \mathrm{O}_{4} /$ PEI-Ac NGs/DOX complexes were dispersed in $1 \mathrm{~mL}$ of phosphate buffer with different $\mathrm{pHs}$ to have a DOX concentration of $1 \mathrm{mg} / \mathrm{mL}$, placed into a dialysis bag with an MWCO of 8000-14000, and immersed into $9 \mathrm{~mL}$ of phosphate buffer at $\mathrm{pH} 7.4$ or $\mathrm{pH}$ 5.5. The whole system was placed in a shaker at a constant temperature of $37^{\circ} \mathrm{C}$. At different time points, $1 \mathrm{~mL}$ of the outside medium was taken out and the absorbance at $480 \mathrm{~nm}$ was measured using a UVvis spectrophotometer. After that, the outer phase was replenished with $1 \mathrm{~mL}$ fresh corresponding buffer solution.

Cell Biological Evaluation and in Vivo Tumor Theranostics. In vitro cytotoxicity assay, flow cytometric assay, and confocal microscopic observation were performed to evaluate the cytotoxicity of the hybrid NGs, the therapeutic efficacy of the NGs/DOX complexes, and the intracellular uptake of the NGs/DOX complexes. All animal experiments were carried out after approval by the ethical committee for animal care of Donghua University and according to the policy of the National Ministry of Health. See additional experimental details in Supporting Information.

\section{ASSOCIATED CONTENT}

\section{SI Supporting Information}

The Supporting Information is available free of charge at https://pubs.acs.org/doi/10.1021/acs.bioconjchem.0c00036.
Additional experimental details, and additional data for hydrodynamic size and zeta potential, drug loading content and drug loading efficiency, colloidal stability assessment, flow cytometry and ICP-OES analysis of cellular uptake of NGs, in vivo biodistribution, and $\mathrm{H} \& \mathrm{E}$ staining (PDF)

\section{AUTHOR INFORMATION}

\section{Corresponding Authors}

Mingwu Shen - College of Chemistry, Chemical Engineering and Biotechnology, Donghua University, Shanghai 201620, People's Republic of China; () orcid.org/0000-0002-10650854; Email: mwshen@dhu.edu.cn

Xiangyang Shi - Department of Interventional and Vascular Surgery, Shanghai Tenth People's Hospital, Tongji University School of Medicine, Shanghai 200072, People's Republic of China; College of Chemistry, Chemical Engineering and Biotechnology, Donghua University, Shanghai 201620, People's Republic of China; CQM-Centro de Quimica da Madeira, Universidade da Madeira, Campus da Penteada, 9000-390 Funchal, Portugal; $\odot$ orcid.org/0000-0001-6785-6645; Email: xshi@dhu.edu.cn

\section{Authors}

Yu Zou - Department of Interventional and Vascular Surgery, Shanghai Tenth People's Hospital, Tongji University School of Medicine, Shanghai 200072, People's Republic of China; CQMCentro de Quimica da Madeira, Universidade da Madeira, Campus da Penteada, 9000-390 Funchal, Portugal

Du Li - College of Chemistry, Chemical Engineering and Biotechnology, Donghua University, Shanghai 201620, People's Republic of China

Yue Wang - Department of Radiology, Shanghai Songjiang District Central Hospital, Shanghai 201600, People's Republic of China

Zhijun Ouyang - College of Chemistry, Chemical Engineering and Biotechnology, Donghua University, Shanghai 201620, People's Republic of China

Yucheng Peng - College of Chemistry, Chemical Engineering and Biotechnology, Donghua University, Shanghai 201620, People's Republic of China

Helena Tomás - CQM-Centro de Quimica da Madeira, Universidade da Madeira, Campus da Penteada, 9000-390 Funchal, Portugal; (1) orcid.org/0000-0002-7856-2041

Jindong Xia - Department of Radiology, Shanghai Songjiang District Central Hospital, Shanghai 201600, People's Republic of China

João Rodrigues - CQM-Centro de Química da Madeira, Universidade da Madeira, Campus da Penteada, 9000-390 Funchal, Portugal; 이이.org/0000-0003-4552-1953

Complete contact information is available at: https://pubs.acs.org/10.1021/acs.bioconjchem.0c00036

\section{Notes}

The authors declare no competing financial interest.

\section{ACKNOWLEDGMENTS}

This research has been financially supported by the SinoGerman Center for Research Promotion (GZ1505), the National Natural Science Foundation of China (81761148028), and the Science and Technology Commission of Shanghai Municipality (19410740200 and 19XD1400100). 
H.T., J.R. and X.S. also thank the support from FCTFundaçâpara a Ciência e a Tecnologia through the CQM Base Fund - UIDB/00674/2020, and Programmatic Fund UIDP/00674/2020, and by ARDITI-Agência Regional para o Desenvolvimento da Investigação Tecnologia e Inovação, through the project M1420-01-0145-FEDER-000005 - Centro de Química da Madeira - CQM+ (Madeira 14-20 Program).

\section{REFERENCES}

(1) Kong, J., Franklin, N. R., Zhou, C., Chapline, M. G., Peng, S., Cho, K., and Dai, H. (2000) Nanotube Molecular Wires as Chemical Sensors. Science 287, 622-625.

(2) Gong, K., Du, F., Xia, Z., Durstock, M., and Dai, L. (2009) Nitrogen-Doped Carbon Nanotube Arrays with High Electrocatalytic Activity for Oxygen Reduction. Science 323, 760-764.

(3) Zhang, K., Zhang, L. L., Zhao, X., and Wu, J. (2010) Graphene/ Polyaniline Nanofiber Composites as Supercapacitor Electrodes. Chem. Mater. 22, 1392-1401.

(4) Carey, G. H., Abdelhady, A. L., Ning, Z., Thon, S. M., Bakr, O. M., and Sargent, E. H. (2015) Colloidal Quantum Dot Solar Cells. Chem. Rev. 115, 12732-12763.

(5) Swarnkar, A., Marshall, A. R., Sanehira, E. M., Chernomordik, B. D., Moore, D. T., Christians, J. A., Chakrabarti, T., and Luther, J. M. (2016) Quantum Dot-Induced Phase Stabilization of $\alpha$-CsPbI3 Perovskite for High-Efficiency Photovoltaics. Science 354, 92-95.

(6) Dreher, M. R., Simnick, A. J., Fischer, K., Smith, R. J., Patel, A., Schmidt, M., and Chilkoti, A. (2008) Temperature Triggered SelfAssembly of Polypeptides into Multivalent Spherical Micelles. J. Am. Chem. Soc. 130, 687-694.

(7) Xiao, T., Li, D., Shi, X., and Shen, M. (2020) PAMAM Dendrimer-Based Nanodevices for Nuclear Medicine Applications. Macromol. Biosci. 20, 1900282.

(8) Zhu, J. Y., and Shi, X. Y. (2013) Dendrimer-Based Nanodevices for Targeted Drug Delivery Applications. J. Mater. Chem. B 1, 41994211.

(9) Xiong, Z., Shen, M., and Shi, X. (2018) Dendrimer-Based Strategies for Cancer Therapy: Recent Advances and Future Perspectives. Sci. China Mater. 61, 1367-1386.

(10) Sasaki, Y., and Akiyoshi, K. (2010) Nanogel Engineering for New Nanobiomaterials: from Chaperoning Engineering to Biomedical Applications. Chem. Rec. 10, 366-376.

(11) Zou, Y., Li, D., Shen, M., and Shi, X. (2019) PolyethylenimineBased Nanogels for Biomedical Applications. Macromol. Biosci. 19, 1900272.

(12) Xie, J., Lee, S., and Chen, X. (2010) Nanoparticle-Based Theranostic Agents. Adv. Drug Delivery Rev. 62, 1064-1079.

(13) Yoo, D., Lee, J.-H., Shin, T.-H., and Cheon, J. (2011) Theranostic Magnetic Nanoparticles. Acc. Chem. Res. 44, 863-874.

(14) Lammers, T., Aime, S., Hennink, W. E., Storm, G., and Kiessling, F. (2011) Theranostic Nanomedicine. Acc. Chem. Res. 44, 1029-1038.

(15) Li, Y., Maciel, D., Rodrigues, J., Shi, X., and Tomás, H. (2015) Biodegradable Polymer Nanogels for Drug/Nucleic Acid Delivery. Chem. Rev. 115, 8564-8608.

(16) Ma, K., Xing, R., Jiao, T., Shen, G., Chen, C., Li, J., and Yan, X. (2016) Injectable Self-Assembled Dipeptide-Based Nanocarriers for Tumor Delivery and Effective in Vivo Photodynamic Therapy. ACS Appl. Mater. Interfaces 8, 30759-30767.

(17) Yan, X., Cui, Y., He, Q., Wang, K., and Li, J. (2008) Organogels Based on Self-Assembly of Diphenylalanine Peptide and Their Application to Immobilize Quantum Dots. Chem. Mater. 20, 15221526.

(18) Yan, X., Zhu, P., and Li, J. (2010) Self-Assembly and Application of Diphenylalanine-Based Nanostructures. Chem. Soc. Rev. 39, 1877-1890.

(19) Sun, W., Yang, J., Zhu, J., Zhou, Y., Li, J., Zhu, X., Shen, M., Zhang, G., and Shi, X. (2016) Immobilization of Iron Oxide
Nanoparticles within Alginate Nanogels for Enhanced MR Imaging Applications. Biomater. Sci. 4, 1422-30.

(20) Zhang, C., Gau, E., Sun, W., Zhu, J., Schmidt, B. M., Pich, A., and Shi, X. (2019) Influence of Size, Crosslinking Degree and Surface Structure of Poly(N-vinylcaprolactam)-Based Microgels on Their Penetration into Multicellular Tumor Spheroids. Biomater. Sci. 7, $4738-4747$.

(21) Ailincai, D., Peptanariu, D., Pinteala, M., and Marin, L. (2019) Dynamic Constitutional Chemistry Towards Efficient Nonviral Vectors. Mater. Sci. Eng., C 94, 635-646.

(22) Laschewsky, A. (2014) Structures and Synthesis of Zwitterionic Polymers. Polymers 6, 1544-1601.

(23) Hess, M., Jones, R. G., Kahovec, J., Kitayama, T., Kratochvíl, P., Kubisa, P., Mormann, W., Stepto, R., Tabak, D., and Vohlídal, J. (2006) Terminology of Polymers Containing Ionizable or Ionic Groups and of Polymers Containing Ions (IUPAC Recommendations 2006). Pure Appl. Chem. 78, 2067-2074.

(24) Zhu, J., Sun, W., Zhang, J., Zhou, Y., Shen, M., Peng, C., and Shi, X. (2017) Facile Formation of Gold-Nanoparticle-Loaded gamma-Polyglutamic Acid Nanogels for Tumor Computed Tomography Imaging. Bioconjugate Chem. 28, 2692-2697.

(25) Sun, W., Zhang, J., Zhang, C., Zhou, Y., Zhu, J., Peng, C., Shen, M., and Shi, X. (2018) A Unique Nanogel-Based Platform for Enhanced Dual Mode Tumor MR/CT Imaging. J. Mater. Chem. B 6, 4835-4842.

(26) Zhu, J., Peng, C., Sun, W., Yu, Z., Zhou, B., Li, D., Luo, Y., Ding, L., Shen, M., and Shi, X. (2015) Formation of Iron Oxide Nanoparticle-Loaded $\gamma$-Polyglutamic Acid Nanogels for MR Imaging of Tumors. J. Mater. Chem. B 3, 8684-8693.

(27) Ibrahim, H., Bindschaedler, C., Doelker, E., Buri, P., and Gurny, R. (1992) Aqueous Nanodispersions Prepared by a Salting-Out Process. Int. J. Pharm. 87, 239-246.

(28) Sun, W., Thies, S., Zhang, J., Peng, C., Tang, G., Shen, M., Pich, A., and Shi, X. (2017) Gadolinium-Loaded Poly(N-vinylcaprolactam) Nanogels: Synthesis, Characterization, and Application for Enhanced Tumor MR Imaging. ACS Appl. Mater. Interfaces 9, 3411-3418.

(29) Battaglia, L., Trotta, M., Gallarate, M., Carlotti, M. E., Zara, G. P., and Bargoni, A. (2007) Solid Lipid Nanoparticles Formed by Solvent-in-Water Emulsion-Diffusion Technique: Development and Influence on Insulin Stability. J. Microencapsulation 24, 672-684.

(30) Lv, F.-F., Zheng, L.-Q., and Tung, C.-H. (2005) Phase Behavior of the Microemulsions and the Stability of the Chloramphenicol in the Microemulsion-Based Ocular Drug Delivery System. Int. J. Pharm. 301, 237-246.

(31) Qi, C., Chen, Y., Huang, J. H., Jin, Q. Z., and Wang, X. G. (2012) Preparation and Characterization of Catalase-loaded Solid Lipid Nanoparticles Based on Soybean Phosphatidylcholine. J. Sci. Food Agric. 92, 787-793.

(32) Desgouilles, S., Vauthier, C., Bazile, D., Vacus, J., Grossiord, J.L., Veillard, M., and Couvreur, P. (2003) The Design of Nanoparticles Obtained by Solvent Evaporation: a Comprehensive Study. Langmuir 19, 9504-9510.

(33) Meurer, R. A., Kemper, S., Knopp, S., Eichert, T., Jakob, F., Goldbach, H. E., Schwaneberg, U., and Pich, A. (2017) Biofunctional Microgel-Based Fertilizers for Controlled Foliar Delivery of Nutrients to Plants. Angew. Chem., Int. Ed. 56, 7380-7386.

(34) Peng, H., Huang, X. B., Melle, A., Karperien, M., and Pich, A. (2019) Redox-Responsive Degradable Prodrug Nanogels for Intracellular Drug Delivery by Crosslinking of Amine-Functionalized Poly(N-vinylpyrrolidone) Copolymers. J. Colloid Interface Sci. 540, 612-622.

(35) Song, C., Sun, W., Xiao, Y., and Shi, X. (2019) Ultrasmall Iron Oxide Nanoparticles: Synthesis, Surface Modification, Assembly, and Biomedical Applications. Drug Discovery Today 24, 835-844.

(36) Ma, D., Shi, M., Li, X., Zhang, J., Fan, Y., Sun, K., Jiang, T., Peng, C., and Shi, X. (2020) Redox-Sensitive Clustered Ultrasmall Iron Oxide Nanoparticles for Switchable T2/T1-Weighted Magnetic Resonance Imaging Applications. Bioconjugate Chem. 31, 352-359. 
(37) Xiong, Z., Shen, M., and Shi, X. (2019) Zwitterionic Modification of Nanomaterials for Improved Diagnosis of Cancer Cells. Bioconjugate Chem. 30, 2519-2527.

(38) Ma, D., Chen, J. W., Luo, Y., Wang, H., and Shi, X. Y. (2017) Zwitterion-Coated Ultrasmall Iron Oxide Nanoparticles for Enhanced T-1-Weighted Magnetic Resonance Imaging Applications. J. Mater. Chem. B 5, 7267-7273.

(39) Luo, Y., Yang, J., Yan, Y., Li, J., Shen, M., Zhang, G., Mignani, S., and Shi, X. (2015) RGD- Functionalized Ultrasmall Iron Oxide Nanoparticles for Targeted $T_{1}$-Weighted MR Imaging of Gliomas. Nanoscale 7, 14538-14546.

(40) Lu, S., Li, X., Zhang, J., Peng, C., Shen, M., and Shi, X. (2018) Dendrimer-Stabilized Gold Nanoflowers Embedded with Ultrasmall Iron Oxide Nanoparticles for Multimode Imaging-Guided Combination Therapy of Tumors. Adv. Sci. 5, 1801612.

(41) Li, J., Hu, Y., Sun, W., Luo, Y., Shi, X., and Shen, M. (2016) Facile Preparation of Hyaluronic Acid-Modified Fe3O4@Mn3O4 Nanocomposites for Targeted T1/T2 Dual-Mode MR Imaging of Cancer Cells. RSC Adv. 6, 35295-35304.

(42) Roca, A. G., Marco, J. F., Morales, M. D. P., and Serna, C. J. (2007) Effect of Nature and Particle Size on Properties of Uniform Magnetite and Maghemite Nanoparticles. J. Phys. Chem. C 111, 18577-18584.

(43) Sun, W. J., Zhang, J. L., Zhang, C. C., Wang, P., Peng, C., Shen, M. W., and Shi, X. Y. (2018) Construction of Hybrid Alginate Nanogels Loaded with Manganese Oxide Nanoparticles for Enhanced Tumor Magnetic Resonance Imaging. ACS Macro Lett. 7, 137-142.

(44) Zhou, B., Zhao, L., Shen, M., Zhao, J., and Shi, X. (2017) A Multifunctional Polyethylenimine-Based Nanoplatform for Targeted Anticancer Drug Delivery to Tumors in Vivo. J. Mater. Chem. B 5, $1542-1550$.

(45) Wang, Y., Cao, X. Y., Guo, R., Shen, M. W., Zhang, M. G., Zhu, M. F., and Shi, X. Y. (2011) Targeted Delivery of Doxorubicin into Cancer Cells using a Folic Acid-Dendrimer Conjugate. Polym. Chem. 2, 1754-1760. 